\title{
Publisher Correction: Direct imaging of the electron liquid at oxide interfaces
}

Kyung Song, Sangwoo Ryu, Hyungwoo Lee, Tula R. Paudel (1), Christoph T. Koch, Bumsu Park (D), Ja Kyung Lee, Si-Young Choi, Young-Min Kim, Jong Chan Kim, Hu Young Jeong (1D, Mark S. Rzchowski, Evgeny Y. Tsymbal, Chang-Beom Eom and Sang Ho Oh

Correction to: Nature Nanotechnology https://doi.org/10.1038/s41565-017-0040-8, published online 5 February 2018.

In the version of this Letter originally published, in two instances in Fig. 1 the layers in the cross-sectional view of the (001) interface were

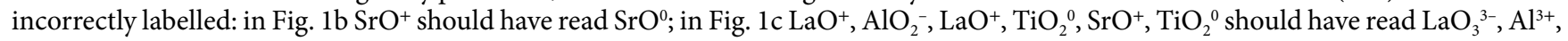
$\mathrm{LaO}_{3}{ }^{3-}, \mathrm{Ti}^{4+}, \mathrm{SrO}_{3}{ }^{4-}, \mathrm{Ti}^{4+}$. In Fig. $3 \mathrm{c}$ the upper-right equation read $-\sigma_{\mathrm{s}}=-e / 2 a^{2}$ but should have read $-\sigma_{\mathrm{s}}=e / 2 a^{2}$ and in Fig. $3 \mathrm{f}$ the lowerright equation read $-\sigma_{\mathrm{s}}=-e / 2 \sqrt{3} a^{2}$ but should have read $\sigma_{\mathrm{s}}=-e / 2 \sqrt{3} a^{2}$. In addition, in the HTML version the affiliation 'Department of Materials Science and Engineering, Pohang University of Science and Technology (POSTECH), Pohang, Republic of Korea' was mistakenly listed as the present address of S.-Y.C. but should have been listed as an affiliation for K.S., B.P., J.K.L., S.-Y.C. and S.H.O. And, in the Supplementary Information file, the text 'small compared with the' was mistakenly added to the beginning of the title. These errors have now been corrected in the online version of the Letter.

Published online: 30 May 2018

https://doi.org/10.1038/s41565-018-0137-8

\section{Publisher Correction: Nanobiophotonics: Nature-inspired sensors}

\section{Wolfgang Fink}

Correction to: Nature Nanotechnology https://doi.org/10.1038/s41565-018-0164-5, published online 6 June 2018.

In the version of this News and Views article originally published, in the author's e-mail address, the domain was incorrectly given as 'e-mail.arizona.edu'; it should have read 'email.arizona.edu'. This has now been corrected in the online versions. 